\title{
The Effect of Reasoning Logics on Real-Time Decision Making
}

\author{
Giampiero E. G. Beroggi, Member, IEEE, and William A. Wallace, Senior Member, IEEE
}

\begin{abstract}
The advent of satellite tracking and communications technology has motivated organizations to investigate centralized real-time support for remote and mobile units and operations. Transportation companies have begun to use satellite tracking systems, production and energy plants are employing intelligent monitoring systems, and on-site emergency managers are being supported by headquarters in their assessment and decision making process. Although different reasoning logics have been proposed for real-time decision making, little attention has been given to the comparative assessment of these approaches. This paper presents empirical evidence on the impact of alternative reasoning logics on individual decision making in real-time. Following the definition of the decision tasks for hazardous operations, commonly used reasoning logics are discussed. Then, results of an empirical comparison of different reasoning approaches are presented. The data were gathered during an experiment with experienced operators. The conclusions of this research are that the selection of the appropriate reasoning logic in support of real-time decision making is crucial.
\end{abstract}

Index Terms-Accuracy, decision support systems, effort, operational risk management, time pressure.

\section{INTRODUCTION}

$\mathbf{R}^{\mathrm{n}}$ EAL-TIME decision making requires tradeoffs between conflicting criteria, such as costs and benefits, under time constraints. The assessment of these criteria, under different scenarios, is done strategically to provide guidelines and procedures (i.e., courses of action) for the decision makers and operators. Optimal courses of action can then be computed algorithmically, where a course of action consists of a temporal ordered sequence of decisions and concomitant selected activities [1]. However, sudden onset events, for which no appropriate response can be devised strategically, can affect the successful completion of these planned courses of action.

Real-time control and decision making has been studied for hazardous operations, such as command and control [2]; transportation of hazardous materials [3]; emergency management [4], and rail operations [5]. It can be generalized as identifying any potential for immediate change in current conditions (monitoring), comparing both to a desired state (assessment), and if there is a gap, taking action to bring the present or future state in concordance with the desired state (choice) [2].

Manuscript received February 5, 1996; revised February 14, 1996 and January 30, 1997.

G. E. G. Beroggi is with Systems Engineering, Policy Analysis, and Management, Delft University of Technology, 2600 GA Delft, The Netherlands.

W. A. Wallace is with Decision Sciences and Engineering Systems, Center for Industrial Innovation, Rensselaer Polytechnic Institute, Troy, NY 12180 USA (e-mail: wallaw@ @pi.edu).

Publisher Item Identifier S 1083-4427(97)07013-6.
For a dispatcher of hazardous material vehicles, monitoring refers to observing the movement of the vehicles on an electronic map. Whenever an unexpected sudden onset event, a real-time event (RTE), occurs, the dispatcher must assess its impact on safety and transportation costs. Examples of RTE's are snow storms and traffic accidents. Then, the "optimality" of the routes for those vehicles that are affected by the RTE (i.e., the vehicles that plan to drive through the area affected by the RTE) must be analyzed. The dispatcher can then decide for all the affected vehicles, i) to leave them on the planned route (i.e., not to change the original course of action), ii) to reroute to other routes (i.e., to search for new courses of action), or iii) to stop the operations.

Real-time decision making must be performed under time pressure and uncertainty. In addition, the alternative courses of action, such as the different roads between origin and destination, are usually represented only implicitly as a graph (e.g., on a map). Explicit courses of action (e.g., the feasible roads between two points on the road network) must first be constructed using some heuristic and an algorithm. Thus, the combinatorial complexity of constructing feasible courses of action (e.g., roads) adds significantly to the overall complexity of the decision situation.

Past research has investigated the limitations in human information processing capabilities in real-time decision situations and concluded that models of the human-machine interaction have to take into account the cognitive aspects of human behavior [6]. We can therefore summarize the characteristics of real-time decision situations for hazardous operations as: time pressure to make decisions, uncertainty, combinatorial complexity, and human limitations in information processing. Therefore, a human-machine system for supporting real-time decision making must strive for an effective sharing of tasks between the human operator and the computer. Our approach is to have the computer perform the computationally intensive tasks, and the operator the cognitive assessments.

\section{Decision Making AND Reasoning APPROACHES IN A REAL TIME ENVIRONMENT}

Real-time decision making is a dynamic process. Decision situations occur at a changing rate and with different task complexity. The literature distinguishes three basic approaches in decision support for dynamic settings. These are outcome feedback, feedforward and cognitive feedback [7]. Outcome feedback turns out to be the most ineffective approach, as shown in studies with a fire-fighting simulation system [8]. Reported reasons are time delay, insufficient information feed- 
back, and inability to make correct conclusions from observed outcome. Cognitive feedback (i.e., provide information to the decision maker about various relations in the system) is the most effective approach, outperforming feedforward (i.e., provide the decision maker with a model of the task prior to performing the task) [7].

In using cognitive feedback, a distinction between presenting information by alternatives and by attributes can be made. Decision making by alternative amounts to providing the decision maker information about feasible alternative courses of action (e.g., routes). In this case, the computation of feasible courses of action is done using algorithms and heuristics. Decision making by attribute amounts to providing the operator information about relations between the impact of the RTE's and the feasibility (and optimality) of alternative courses of action.

There are various examples of reasoning logics for generating feasible and optimal courses of action (i.e., routes) for hazardous operations. The most basic approach is to let the operator determine such courses of action with no computer support (i.e., using only his/her individual reasoning logic). The only support that is provided to the operator is a graphical, visual interactive display that facilitates the construction of courses of action. Such a reasoning model is called visual interactive model, VI model.

A more sophisticated approach is to use a simple heuristic to compute courses of action. For hazardous operations, it is quite common to adopt a conservative approach, i.e., to assume that all activities that are affected by a RTE have become too risky to employ. This model is called conservative heuristic model, CH model. These two reasoning approaches pertain to decision making by alternative because they help the operator decide on new courses of action by focusing on feasible alternatives.

Many reasoning approaches are based on multi-attribute utility theory (MAUT) and mathematical programming. A common approach is to evaluate every activity with a given set of criteria. Hazardous operations, such as the transportation of dangerous goods, are usually valued using the criteria risk and costs. To compute optimal routes, risks and costs of every road segment must be evaluated. Risks are then transformed into costs and added to the transportation costs, resulting in total risk-costs. The optimal paths between any two origins and destinations is the one with minimum total risk-cost values, subject to physical constraints. Tradeoff values needed to transform risks to costs can be found in the literature [9].

The mathematical program for the multicriteria route optimization model is then the following, where $r c_{i}$ are the total risk-costs for the road segment $i$, and $x_{i j}$ is the road segment from node $i$ to node $j$ : minimize $\Sigma x_{i} r c_{i}$, subject to $\Sigma_{j} x_{i j}-\Sigma_{k} x_{k i}=\{1$ for $i=$ origin, -1 for $i=$ destination, an 0 for all other $i\}, x_{i j}=0$ or 1 for all $i$. Note that the constraints refer only to physical characteristics of the network. A reasoning approach based on such a model is called a multi-attribute utility model, MAU model.

Despite the fact that many MAU models have been proposed for real-time decision support, they have been criticized for not taking into account humans' limitations in information processing, particularly their inability to apply probability logic [10]. Consequently, other reasoning approaches have been proposed and employed for real-time decision support of large scale operations, e.g., fuzzy set theory and approximate reasoning [11], genetic algorithms [12], and lexicographic goal programming [4].

The latter approach is based on a set of cognitive assumptions about the human operator performing in a real-time environment. These assumptions consider i) human's cognitive limitations, ii) that uncertainties in real-time decision situations are more difficult to assess than in strategic settings, and iii) that searching for suboptimal decisions can be more efficient than searching for optimal solutions. The cognitive assumptions are operationalized as a preference algebra which leads to the lexicographic goal programming approach, i.e., the optimal course of action is the one that scores best on the least preferred criteria.

The reasoning principle of the search algorithm that constructs the optimal course of action in this decision situation is the following. It fans out from the origin decision toward the destination decision by adding new activities (e.g., road segments) according to the following priorities: 1) it never takes a road segment that must be avoided (too high risk); 2) it avoids as much as possible road segments with high risks; 3 ) it minimizes transportation costs; 4 ) it avoids as much as possible road segments with low risks. For a detailed discussion of the algorithm see [13].

Thus, risks are traded off against costs in the sense that high risks must be avoided no matter the costs that are involved, and low risks are only avoided if no additional costs are involved. In addition, risks can be so high so that the road segment cannot be used. A reasoning approach based on such a model is called ordinal preference model, $O P$ model.

\section{EMPIRICAL ASSESSMENT OF REASONING APPROACHES}

Reasoning logics imbedded into decision support systems should be assessed empirically. The most interesting measures of performance are effort and accuracy [14]. Effort refers to the time it takes the operator to make decisions, the cognitive strain to make decisions, and the effort involved in using the decision support system (DSS). Accuracy refers to the quality of the decisions.

Variables affecting effort and accuracy are the reasoning logic, task complexity, cognitive load, training and experience of the operator. The reasoning logics of the VI, $\mathrm{CH}$, OP, and MAU models have been implemented into a DSS for dispatchers of hazardous material shipments for the Capital District region of New York State [15], and for Switzerland [3].

The latter DSS was used to simulate different task situations for empirical assessment of the four reasoning logics. Significant insights can be gained if the DSS can simulate the work environment of operators using advanced technology. To achieve this, the DSS was developed in a multimedia environment using graphics, text, drawing, animation, and voice output. Operators only use the mouse as input device. The simulated task situations can be repeated with different scenarios. Tests confirmed that computer knowledge did not 


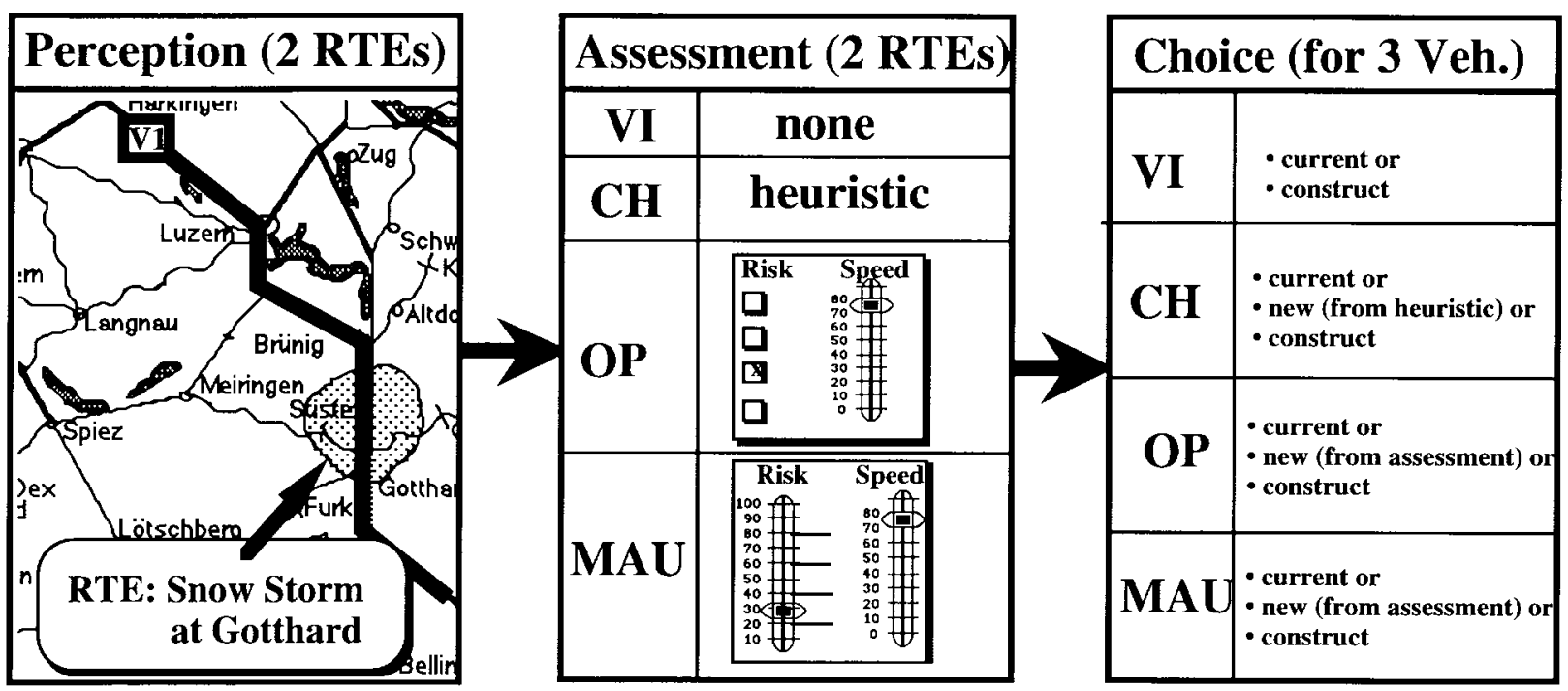

Fig. 1. Decision making processes for the four reasoning approaches.

affect the results, and learning during the assessment process did not occur.

\section{A. Assessment in a Simulated Work Environment}

An assessment of the four reasoning logics was done with 32 experienced dispatchers at a dispatching school in Wil, Switzerland. Three scenarios were designed to simulate decision situations typical of a dispatcher of vehicles carrying hazardous materials. In every scenario, the dispatcher could monitor the movement of three vehicles using a computer generated map. While the vehicles were moving on the map, two RTE's occur that affected safety and costs of some of the shipments. The subjects must then make rerouting decisions for all three vehicles. These decision situations are repeated with three slightly different scenarios, yielding a maximum of 72 rerouting decisions for each reasoning model (nine per subject). In addition, the times for making these decisions were also recorded.

While the means to communicate the occurrence of the RTE's (see Fig. 1: perception) is the same for all four models, the remaining components of the decision making process (see Fig. 1: assessment and choice) differ among the four models. Subjects working with the OP and the MAU models must first assess the impact of the RTE's on risk and cost (assessment). For subjects working with the $\mathrm{CH}$ model, the system automatically computes an alternate route for all vehicles based on the conservative heuristic that all RTE's increase the risk such that they must be avoided. Subjects working with the VI model had no reasoning support in finding new routes. RTE's were designed to be either hazardous or harmless. Hazardous RTE's increased the risks significantly, while harmless RTE's did not.

After the assessment phase, all the subjects had to make choices for all three vehicles (choice). Subjects with the VI model would only see the current routes of the vehicles. New routes could be selected by clicking on the appropriate road segments with the computer mouse. Subjects with the other models saw, in addition to the current routes, new routes that result from the assessments or from the heuristic. Their choice was between staying on the current route, taking the new route, or constructing a new route.

Fig. 1 summarizes the decision making process consisting of perception, assessment, and choice. It can be seen that MAU subjects used a slide bar to assess the risks, while OP subjects used risk classes. Stress was created by limiting the decision time which was shown to the subjects by a timer at the top boarder of the screen.

The discussion of the results concerning the comparison of the four models for effort and accuracy are reported in [16]. Assessing the effects of RTE's with the ordinal preference structure (OP model), prior to making routing decisions, was found to be significantly more efficient and accurate than searching directly for new routes. Using a numeric scale to assess safety and cost in cases of a RTE was disliked by the subjects and led to inferior results.

In the following sections we discuss the results that refer to the effects that effort and task complexity had on the accuracy of the decisions within and across the four models. Furthermore, findings on task sharing between humans and DSS are also reported. Prior to these discussions, the role of the DSS on these results is addressed. This is important because the purpose was to assess different reasoning logics and not the DSS.

\section{B. The Decision Support System}

When assessing reasoning logics embedded into DSS's, one must be careful to separate the effects of the reasoning logics from those of the DSS. The purpose of the experiment was to assess the effects of the reasoning and logics and not those of the DSS. Thus, the effects of the DSS should be eliminated, or at least be the same for the four systems.

Effects of the DSS on the results of the experiment can stem from various sources. Since four slightly different DSS's were used for the four reasoning logics, effects across the 
DSS's, as well as within the system, had to be eliminated. Effects within the system refer to the learning effect that might occur during the experiment when working with rather complex DSS's that require certain skills for effective use. Effects across the systems refer to inevitable, although minor, design differences among the four systems.

To make sure that the results, as reported in the following sections, are not affected by the DSS's, structured questions were posed to the subjects, i.e., the purpose of asking these questions was to help insure that the results refer to the differences in the reasoning logics and not to the DSS('s). The responses to the questionnaires show that both the "ease of using the DSS" as well as "sufficiency of training before the experiment" scored in the average 8.8 and 8.6, respectively, on a scale from one (very false) to ten (very true), with no significant differences among the four DSS's. Moreover, "clearness of simplification" (7.9) and "clearness of scenarios" (8.9) where high, while "confusion during scenarios" (3.9) was low, which supports the conclusion that the DSS's had no significant effect on the results. Thus, we could conclude that from the point of view of the subjects there was no effect within (no learning) and across (no cross effects) the DSS's.

It was of course the intention to eliminate the effects of the DSS in the design phase. For this purpose, the DSS's had to be as similar as possible and very easy to use. Therefore, the DSS's were build in a multimedia environment. Information was given to the subjects in form of voice output (instructions about movement of vehicles and announcement of events), graphs (map with road network), text (in addition to the voice output), and animation (moving vehicles, drawing of routes). Input was done solely with the computer mouse. It was interesting to note that the subject who performed best had no previous computer experience.

To eliminate effects across the DSS's, the four DSS's were adapted from one main DSS. Only absolutely necessary changes referring to the characteristics of the four approaches were made. These refer to the input devises for risk and cost assessment. Everything else, including the layout of the scenarios, the time the events occur, the way the information is presented to the subjects, etc., was completely identical among the four DSS's. In fact, the main body of the DSS's uses the same computer code. From a design point of view, everything possible was done to eliminate effects within the DSS's (by using a multimedia environment) and across the DSS's (by adapting the four DSS's from one core DSS).

\section{Effort and Accuracy}

Accuracy is measured as the number of correct decisions. A rerouting decision is considered "correct" if the route is both safest and minimum cost (i.e., fastest); it is considered "acceptable" if the route avoids a hazardous RTE but is not the fastest one, else it is considered "wrong," Since every subject had to make nine rerouting decisions, the sum of "correct," "acceptable," and "wrong" decisions is nine. For example, a possible score is five correct, two acceptable, and two wrong decisions $(5 \mathrm{c} / 2 \mathrm{a} / 2 \mathrm{w})$, for a total of nine decisions. Fig. 2 shows the relation between accuracy (scores) and effort (decision

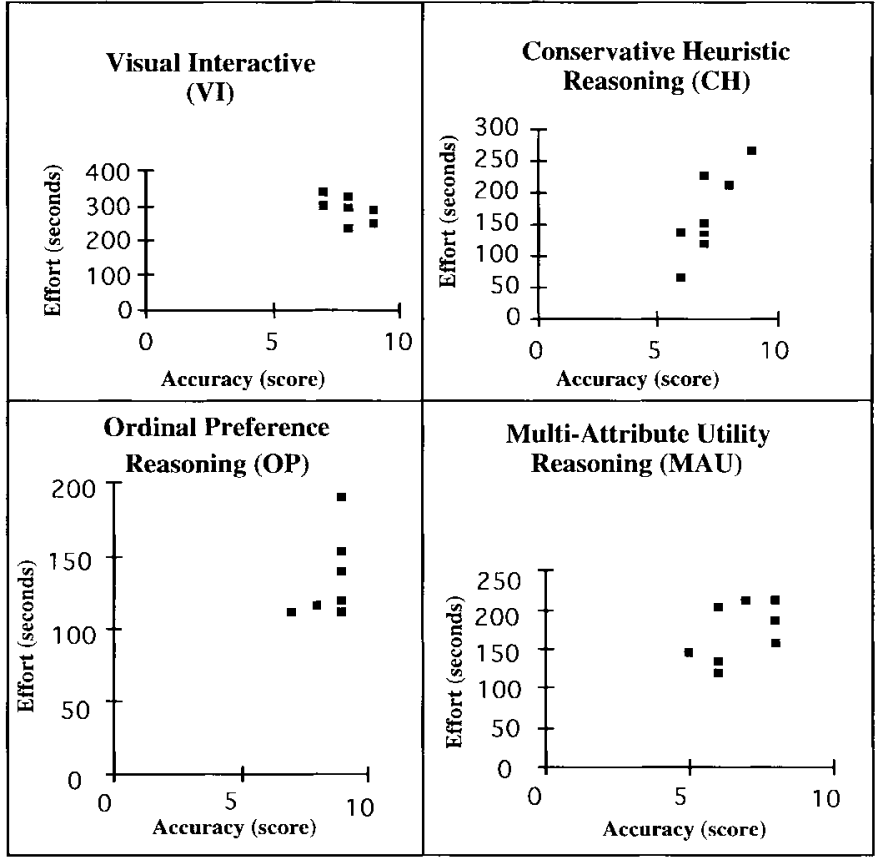

Fig. 2. Accuracy (score: correct plus acceptable decisions) versus effort (decision time) for the four reasoning approaches.

time) for the eight subjects per model, where score is defined as the sum of correct plus acceptable decisions.

The $\mathrm{CH}$ and MAU models show a positive correlation between effort and accuracy (Fig. 2), i.e., the more time the subjects invested in decision making, the higher the score. The VI model shows a negative correlation. This stems from the fact that constructing new routes by clicking with the computer mouse on the appropriate road segments yields higher scores (accuracy) but also takes more time (effort). The relation between effort and accuracy for the OP model seems to be different. Low scores were obtained where decisions were made rapidly. The best scores (i.e., all nine solutions are correct) were obtained if subjects invested at least more than $120 \mathrm{~s}$ (maximum time available for each subject is $360 \mathrm{~s}$ ). These results are statistically significant at the $5 \%$ level for the $\mathrm{CH}$ model, and at the $10 \%$ level for the VI model (omitting one outlier), while the results for the OP and MAU models represent merely trends.

\section{Task Complexity and Accuracy}

Task complexity can be defined in different ways. Since the number of vehicles to be rerouted and the number of RTE's were constant for all three scenarios, task complexity can be defined as the type of RTE (hazardous or harmless) and number of RTE's that affected the vehicles. Of the two RTE's that occurred in each scenario, one did increase the risk (hazardous) and the other did not (harmless). For every scenario, one vehicle was affected by the hazardous RTE, another by the harmless RTE, and the third by both RTE's. The vehicles that were affected by the corresponding RTE's were determined randomly (for all the subjects the same). The most complex rerouting decisions of the three, is the one where the vehicle is affected by both RTE's. For the other 
two decisions, task complexity is assumed to be higher when effort (time) to assess the RTE is higher. The OP subjects took $11.2 \pm 3.5 \mathrm{~s}$ to assess the harmless RTE and $10.8 \pm 2.9 \mathrm{~s}$ to assess the hazardous RTE. The MAU subjects took $12.1 \pm$ $2.9 \mathrm{~s}$ to assess the harmless RTE and $15.2 \pm 4.6 \mathrm{~s}$ to assess the hazardous RTE. Since there is no significant difference in the RTE assessment times for both models, we conclude that the assessment of a harmless or a hazardous RTE is of the same task complexity. The effort to choose a route for the three vehicles were not recorded; only the overall choice times for all three vehicles per scenario were recorded. The choice times per vehicle could have given insights about the relation between task complexity and effort (e.g., more complex tasks might require more effort to solve).

Table I shows the relation between accuracy (scores for correct/acceptable/wrong rerouting decisions) and task complexity. It can be seen that accuracy is lowest where vehicles were affected by both RTE's. This result holds for all models except for the OP model. Accuracy for the OP model seems to be independent of task complexity. When we consider only "correct" solutions, the VI and the $\mathrm{CH}$ models also show a decrease in accuracy between hazardous and harmless RTE. Accuracy with the VI, $\mathrm{CH}$, and MAU models decreases with increasing task complexity, while again the OP model seems not to be affected by increased task complexity.

Interesting to note is that $\mathrm{CH}$ subjects had a very low score for vehicles that were affected by both RTE's. An explanation is that for the vehicles that were affected by both RTE's, both the current route and the new route were wrong. For the vehicle that is affected only by the harmless RTE, the correct route is identical with the original route. For the third vehicle, which is affected only by the hazardous RTE, the correct route is the one computed with the conservative heuristic.

The daily work of operators involves higher task complexity and consequently more time pressure than simulated in this experiment. For example, a dispatcher of vehicles carrying hazardous materials must handle more vehicles, and RTE's occur more frequently. The most appropriate reasoning approach seems to be the OP model. It is based on a lexicographic reasoning approach which i) provides more support than the VI model, ii) is more flexible than the $\mathrm{CH}$ model, and iii) is more conservative than the MAU model. In fact, it has been found that decision makers are less risky (more conservative) under time pressure [17].

\section{E. Cognitive Load}

Cognitive load can be characterized by the effort expanded on assessment and choice. Table II shows the efforts (times in seconds) for assessment and choice for the $\mathrm{CH}$, OP, and MAU reasoning logics. Given are the average times (efforts) for decision making for those scenarios where the subjects made no mistakes ("no-mistake scenarios," i.e., both assessments and choices are correct), and the average times (efforts) of the other scenarios (i.e., some mistakes were made). Note that VI subjects could not assess the RTE's and had no new routes proposed by the DSS. The assessment times for the $\mathrm{CH}$ model are zero, since the assessments are done automatically with a conservative heuristic.
TABLE I

ACCURACY (CORRECT/ACCEPTABLE/WRONG DECISIONS) AND Task Complexity (Harmless, HaZardous, and Both RTE's)

\begin{tabular}{|c|c|c|c|}
\hline Model & $\begin{array}{l}\text { Scores for } V \\
\text { harmless RTE }\end{array}$ & $\begin{array}{l}\text { ehicles that were } \\
\text { hazardous RTE }\end{array}$ & $\begin{array}{l}\text { affected by: } \\
\text { both RTEs }\end{array}$ \\
\hline VI & $19 / 1 / 4$ & $17 / 6 / 1$ & $14 / 8 / 2$ \\
\hline $\mathrm{CH}$ & $24 / 0 / 0$ & $22 / 1 / 1$ & $8 / 2 / 14$ \\
\hline $\mathrm{OP}$ & $23 / 0 / 1$ & $21 / 2 / 1$ & $23 / 0 / 1$ \\
\hline MAU & $17 / 0 / 7$ & $22 / 0 / 2$ & $12 / 3 / 9$ \\
\hline
\end{tabular}

The efforts for the "no-mistake" scenarios can be used to assess the effects of the reasoning logic on correct solutions. The differences between the OP and MAU models for both the average assessment times and the variances are not significant. Thus, there is no benefit in using either reasoning logic in regard to the assessment-effort. On the contrary, the dispatcher must spend more time when using the OP and MAU models compared to the $\mathrm{CH}$ model which does the assessment automatically.

For the choice phase, the $\mathrm{CH}$ model is counterproductive. Choice-effort in "no-mistake" scenarios is significantly greater than in the other scenarios. A possible reason for this is that $\mathrm{CH}$ subjects realized that many routes proposed by the conservative heuristic were wrong and they spent time to determine the correct route.

The variances for choice-times are for all three reasoning approaches significantly smaller for "no-mistake" scenarios than for the other scenarios. This means that the efforts spent on wrong choices vary more than the efforts spent on correct choices. A possible explanation is that wrong choices were obtained either by rapid, random selection of one of the two routes proposed by the system, or by spending time fruitlessly in searching for the correct solution.

Two of the three consecutive tasks that were part of this decision process are assessment followed by choice (as shown in Fig. 1). The OP model reduces significantly the choiceeffort, while the MAU model does not. Moreover, although the OP and MAU models have no significantly different choiceefforts, the OP model has significantly smaller variances in choice-effort than the MAU model. Therefore, the effort spent on correct decisions ("no-mistake" scenarios) is more stable, i.e., the variances in effort are smaller than for when mistakes are made.

We can postulate that high accuracy (high number of correct decisions) and stable effort (small variance in the effort to obtain the high accuracy) are related to each other, i.e., high accuracy is not completely random (at least less random than low accuracy). There seems to be an optimal amount of effort needed to obtain maximum accuracy; more or less than this effort results in inferior solutions.

From a design perspective, we can conclude that a reasoning logic should not have as one of its goals rapid decision making but should strive for an appropriate balance between amount of decision support and task complexity. Since tasks in realtime decision making may have different task complexities, 
TABLE II

Cognitive Load with OP and MAU Models Measured in Seconds

\begin{tabular}{ll|cll}
\hline & & CH & OP & MAU \\
\hline Effort for & scenarios without mistakes & 0 & $22.3 \pm 4.2$ & $28.1 \pm 7.1$ \\
Assessment & scenarios with some mistakes & 0 & $21.0 \pm 7.2$ & $26.5 \pm 5.6$ \\
\hline Effort for & scenarios without mistakes & $82.6 \pm 14.0$ & $17.3 \pm 3.5$ & $26.8 \pm 7.0$ \\
Choice & scenarios with some mistakes & $40.3 \pm 27.0$ & $41.3 \pm 20.0$ & $30.2 \pm 18.2$ \\
\hline
\end{tabular}

different decision support approaches should be provided. This could mean that a DSS should provide different assessment and choice strategies. In fact, cognitive load can often better be reduced by switching choice strategy than by speeding up the current strategy [18].

\section{F. Final Judgment}

After the dispatchers of the OP and MAU models have assessed the impact of the RTE's on risk and costs, new routes were computed automatically by the system and presented to them for choice. For the $\mathrm{CH}$ model, subjects had nothing to assess since new routes were computed automatically using a conservative heuristic. Therefore, the picture on the screen for the choice phase (see Fig. 1) was the same for the $\mathrm{CH}$, OP, and MAU models. This picture contained the current routes as well as the newly computed routes which were presented for choice. In addition, subjects could decide to construct a completely different route if they decided not to take either the current or the new route.

For the choice process, i.e., the selection of new routes for the three vehicles, $\mathrm{CH}, \mathrm{OP}$, and MAU subjects were presented for every vehicle (one at the time) two routes (the original and the new one) to choose from. Some of the subjects carefully examined these two routes in the context of the geographic display. Less critical subjects would trust the new routes proposed by the system; e.g., some $\mathrm{CH}$ subjects accept the routes generated with the conservative heuristic, and some OP and MAU subjects simply follow their assessment.

Fig. 3 shows the conditional scores of correct plus acceptable solutions versus wrong solutions per model, given that the screen presented the correct route (solution) or given that both routes are not correct. The correct solution was either the new computed route (when the vehicle was affected by a hazardous RTE) or the original route (when the vehicle was affected by a harmless RTE).

OP subjects were presented the correct solution on the screen (either the original route or the new computed route based on their assessment) in $89 \%$ of the choice situations. If they saw the correct route, they never made a "wrong" choice. $\mathrm{CH}$ and MAU subjects were shown the correct route in $67 \%$ and $68 \%$, respectively.

Of special interest are those cases, where the subjects were not presented the correct route on the screen. For these cases, OP subjects more often selected the correct solution (63\%) than the $\mathrm{CH}(42 \%)$ and MAU (35\%) subjects. It is rather surprisingly that MAU subjects had the lowest frequency for "savings" (35\%), i.e., to make the right choice although only wrong solutions are presented for choice.

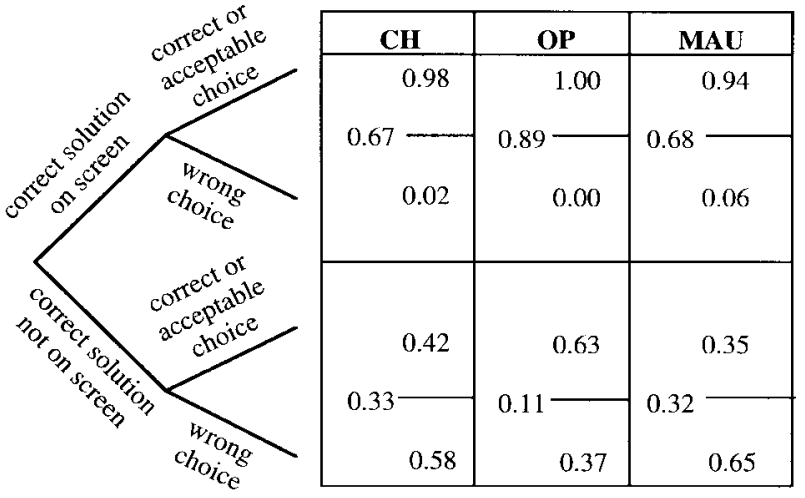

Fig. 3. Marginal frequencies for seeing "correct" solution on the screen, and conditional frequencies for choosing the "wrong" solution.

\section{SUMMARY AND CONCLUSIONS}

Four reasoning approaches in support of real-time decision making have been assessed in a simulated work environment with experienced operators. They range from no reasoning support, to ordinal preference model, to numerical utility model, and finally to automatic assessment with a conservative heuristic. The results show that heuristic and utility reasoning models have a positive correlation between effort and accuracy, while no reasoning support does not. The OP model, which has been found to be the most accurate and to require the least effort, seems to yield high accuracy independent of the decision effort. In addition, the results show that the accuracy of the OP model is not affected by task complexity, while the other models show decreased accuracy with increasing task complexity. Comparing the three reasoning approaches that computed new routes after a RTE $(\mathrm{CH}, \mathrm{OP}$, and MAU model), the OP model creates less cognitive load than the other two models. In addition, the OP model reduces more the cognitive load than the MAU model, while the OP model can be counterproductive. Finally, operators working with the $\mathrm{CH}$ and MAU models seem to accept more easily incorrect results generated by the system than those working with the OP model.

Innovations in communications and computing technology have made possible real-time monitoring and control of hazardous operations. These technological advances are providing the capability for operational decision making. Consequently, alternative reasoning logics have been proposed for supporting the assessment and choice process involved in decision making under uncertainty. It is our contention that the appropriateness of a reasoning logic must be assessed empirically, if possible, in situ or at least in simulated decision situations. In so doing, one must be careful that the reasoning logic is tested and 
not the technology in which it is embedded. Human-machine interfaces that enable one to "see," "hear," and "feel" in a virtual environment do not ensure that the user is "smarter" and better able to make decisions under uncertainty and time pressure.

A conclusion of this research is that the selection of the appropriate reasoning logic in support of real-time decision making is crucial. However, since each subject worked with only one of the four reasoning logics, no conclusions can be drawn whether a combination of the logics could yield better results. In fact, as Hulland and Kleinmuntz [18] note, time pressure does not necessarily force one to employ the current choice strategy more rapidly, but it could cause a shift in choice strategy. For example, if events (such as snow storms) have been perceived to be risky, the conservative heuristic logic would be appropriate; but in instances where dangerous and harmless events are present simultaneously, assessment by attribute seems to be the most appropriate strategy. For noncomplex rerouting situations, the dispatcher might select the new routes without the help of the DSS.

Further research should focus on relaxing some of the stringent constraints that were used in this experimental setting. For example, more vehicles and more events would significantly increase the complexity of the tasks. This would make the scenarios more realistic but also include more parameters requiring a more complex experiment to generate the necessary data.

Despite the fact that more investigations must complement this work before firm conclusions can be drawn, the results of these research can have an impact on the design of human-machine systems and on the tasks of organizations focusing on centralized real-time decision support for remote and mobile units. Efforts in this directions are going on in different domains all over the world. Examples are the U.S. projects relating to the intelligent transport systems (IVTS), the European DRIVE projects addressing issues of advanced road transport telematics, as well as commercially available satellite tracking systems (e.g., Euteltracs and Omnitracs) that have incorporated little decision support so far. However, the results of this research show that the appropriate reasoning approach, embedded into a DSS, can reduce effort and improve accuracy in real-time decision making.

Thus, the steadily growing use of real-time decision making technology by industry (in general regardless of the economic benefits), coupled with the fact that decision makers tend to adapt their decision making strategy to the decision aid that reduces mostly effort (mostly regardless of the accuracy) [19], motivates further investigations of real-time decision support.

\section{REFERENCES}

[1] G. E. G. Beroggi and W. A. Wallace, "A decision logic for operational risk management," in Computational Organization Theory, K. M. Carley and M. J. Prietula, Eds. Hillsdale, NJ: Lawrence Erlbaum, 1994, pp. 289-308.

[2] W. A. Wallace, "Command and control: A team problem solving and decision making process," RADC/COE, Griffiss AFB, NY, 1989.

[3] G. E. G. Beroggi, A. Hersperger, W. A. Wallace, M. Wiedmer, and M. B. Zumsteg, Operationelle Routenwahl im Gefahrenguttransport:
Methodische Ansätze, Computerimplementation, experimentelle Bewertung, Verlag der Fachvereine, Zürich, 1993 (in German).

[4] G. E. G. Beroggi and W. A. Wallace, "Operational risk management: A new paradigm for decision making," IEEE Trans. Syst., Man, Cybern., vol. 24, pp. 1450-1457, Oct. 1994.

[5] R. D. Burns, "Safety and productivity improvement of railroad operations by advanced train control systems," in IEEE/ASME Joint Conf., 1989, pp. 33-38.

[6] G. Mancini, "Commentary: Models of the decision maker in unforseen accidents," Int. J. Man-Mach. Studies, vol. 27, pp. 631-639, 1987.

[7] K. Sengupta and T. K. Abdel-Hamid, "Alternative conceptions of feedback in dynamic decision environments," Manage. Sci., vol. 39, no. 4, pp. 411-428, 1993.

[8] B. Brehmer, "Strategies in real-time, dynamic decision making," in Insights in Decision Making: A Tribute to Hillel J. Einhorn, R. Hogarth, Ed. Chicago, IL: Univ. Chicago Press, 1990.

[9] B. Fischhoff, S. Lichtenstein, P. Slovic, L. Derby, and R. L. Keeney, Acceptable Risk. Cambridge, U.K.: Cambridge Univ. Press, 1981.

[10] A. Tversky and D. Kahneman, "Exceptional vs. intuitive reasoning: The conjunction fallacy in probability judgement," Psychol. Rev., vol. 90 pp. 293-315, 1983

[11] T. Lotan and H. N. Koutsopoulos, "Models for route choice behavior in the presence of information using concepts from fuzzy set theory and approximate reasoning," Transportation, vol. 20, no. 2, pp. 129-155, 1993.

[12] Z. Michalewicz, G. A. Vignauz, and M. Hobbs, "A nonstandard genetic algorithm for the nonlinear transportation problem," ORSA J. Comput., vol. 3, no. 4, pp. 307-316, 1991

[13] G. E. G. Beroggi, "A real-time routing model for hazardous materials," Europ. J. Oper. Res., vol. 75, no. 3, pp. 508-520, 1994.

[14] E. J. Johnson and J. W. Payne, "Effort and accuracy in choice," Manage. Sci., vol. 31, no. 4, pp. 395-414, 1985.

[15] G. E. G. Beroggi and W. A. Wallace, "Real-time control of the transportation of hazardous materials," J. Urban Regional Inf. Syst. Assoc., vol. 4, no. 1, pp. 56-65, 1992.

[16] G. E. G. Beroggi and W. A. Wallace, "Operational control of the transportation of hazardous materials: An assessment of alternative decision models," Manage. Sci., vol. 41, no. 12, pp. 1962-1977, 1995.

[17] H. Ben Zur and S. J. Breznitz. "The effect of time pressure on risky choice behavior," Acta Psychol., vol. 47, pp. 89-104, 1981.

[18] J. S. Hulland and D. N. Kleinmuntz, "Factors influencing the use of internal summary evaluations versus external information in choice," $J$. Behavioral Decision Making, vol. 7, pp. 79-102, 1994.

[19] P. Todd and I. Benbesat, "An experimental investigation of the impact of computer based decision aids on decision making strategies," Inf Syst. Res., vol. 2, no. 2, pp. 87-115, 1991.

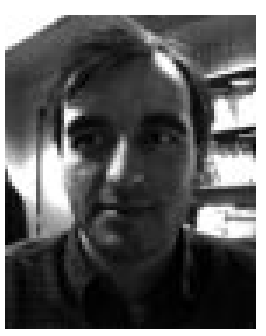

Giampiero E. G. Beroggi (S'91-M'91) received the Ph.D. and M.S. degrees from Rensselaer Polytechnic Institute, Troy, NY, and the Dipl. Ing. from ETH-Zürich, Switzerland.

$\mathrm{He}$ is Associate Professor of Policy Analysis at the School of Systems Engineering, Policy Analysis, and Management, Delft University of Technology, Delft, The Netherlands. His research interests include operational decision support systems, multiattribute spatial decision making, and decision modeling in policy management.

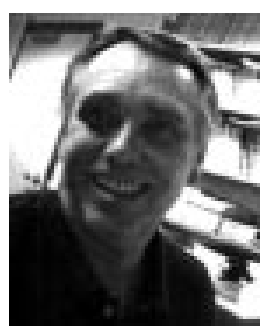

William A. Wallace (M'90-SM'96) is Professor of Decision Sciences and Engineering Systems at Rensselaer Polytechnic Institute, Troy, NY. He has over 20 years experience in research and development in management science and decision support systems, with particular emphasis on crisis management. His was recently appointed Vice-Chair of the National Research Council's Committee on Advanced Information Technology for the Maritime Industry. $\mathrm{He}$ is presently engaged in research on computer-based decision aids for emergency managers, and the process of modeling. 